\title{
Meta
}

Journal des traducteurs

Translators' Journal

\section{Willson, P. (2004) : La Constelación Sur. Traductores y traducciones en la literatura argentina del siglo $X X$, Buenos Aires, Siglo veintiuno editores, 293 p.}

\section{Georges L. Bastin}

Volume 50, numéro 3, août 2005

Le prisme de l'histoire

The History Lens

URI : https://id.erudit.org/iderudit/011618ar

DOI : https://doi.org/10.7202/011618ar

Aller au sommaire du numéro

Éditeur(s)

Les Presses de l'Université de Montréal

ISSN

0026-0452 (imprimé)

1492-1421 (numérique)

Découvrir la revue

Citer ce compte rendu

Bastin, G. L. (2005). Compte rendu de [WILlson, P. (2004) : La Constelación Sur. Traductores y traducciones en la literatura argentina del siglo XX, Buenos Aires, Siglo veintiuno editores, 293 p.] Meta, 50(3), 1060-1062.

https://doi.org/10.7202/011618ar d'utilisation que vous pouvez consulter en ligne.

https://apropos.erudit.org/fr/usagers/politique-dutilisation/ 


\section{Willson, P. (2004): La Constelación Sur. Traductores y traducciones en la literatura argentina del siglo XX, Buenos Aires, Siglo veintiuno editores, 293 p.}

La Constelación Sur est une aventure céleste. D’abord par le sujet, la mise en scène des étoiles de la traduction et de l'édition du $\mathrm{xx}^{\mathrm{e}}$ siècle en Argentine. Ensuite par le style aéré, voire aérien de l'auteure, qui vous transporte dans les cafés de Buenos Aires. J'ajouterais, enfin, par la couleur, celle de l'équipe nationale argentine de football; l'ouvrage transpire littéralement l'Argentine. La voûte de cette aventure est simple mais robuste: cinq chapitres qui nous font explorer 1. la littérature étrangère dans «les librairies populaires»; 2. Victoria Ocampo, la traductrice romantique; 3. Jorge Luis Borges, le traducteur d'avant-garde; 4. José Bianco, le traducteur classique; et 5. La Constellation Sur.

L'auteure détient un doctorat en littérature de l'Universidad de Buenos Aires; à la Faculté de philosophie et lettres de cette université, elle enseigne la littérature argentine, et la traduction littéraire et la théorie de la traduction à l'Instituto Superior en Lenguas Vivas «Juan R. Fernández». En 2003, elle est lauréate du prix du meilleur essai du Fondo Nacional de las Artes. Elle a notamment traduit Paul Ricœur, Jean Starobinski, Luce Irigaray, Slavoj Zizek et Roland Barthes.

Dans une introduction dense mais solidement argumentée, elle démystifie d'abord «la bonne manière de traduire", question qui selon l'auteure ne suscite que des débats byzantins. Elle démystifie ensuite la notion de «littérature nationale, tributaire de conceptions romantiques - voire ingénues - de 'génie artistique,' 'créativité', 'originalité'... » (p. 20). C'est pourquoi elle cherche sa voie interprétative dans la théorie des polisystèmes et l'école de la manipulation. Des trois instances qui coexistent dans tout système littéraire, la production, la tradition et l'importation, elle s'arrêtera à la dernière: la littérature traduite. Willson toutefois ne se réclame d'aucune théorie de la traduction, mais adhère plutôt au courant de la critique littéraire. Elle met donc les outils de la recherche traductologique au service d'une perspective critique. C'est ainsi qu'elle accorde une grande importance aux stratégies de traduction et, pour les décrire, ne répugne pas à procéder à l'analyse comparative sans toutefois l'utiliser à des fins prescriptives.

Patricia Willson, dont l'objectif est d'étudier l'impact de la traduction dans la production littéraire et dans l'industrie de l'édition de l'Argentine de la moitié du $\mathrm{xx}^{\mathrm{e}}$ siècle et dans l'échange symbolique entre cultures différentes, se constitue un corpus: la maison d'édition et revue Sur ainsi que d'autres éditeurs étroitement associés d'une part, et de l'autre trois grands noms de la traduction hispano-américaine: Ocampo, Borges et Bianco.

Dans la plus pure tradition moderne de la critique littéraire et de la sociologie latinoaméricaine représentée notamment par l'«hybridité culturelle» de Nestor García Canclini, la «totalité contradictoire» de Antonio Cornejo Polar et l'anthropophagie de Oswald de Andrade, Patricia Willson choisit délibérément de travailler trois traducteurs, prolifiques, de classiques du $\mathrm{xx}^{\mathrm{e}}$ siècle qui, en outre, se sont exprimés à propos de leur pratique et de leur vision de la traduction. Mais surtout trois traducteurs qui se situent inégalement sur le continuum de la littéralité à la liberté et qui font partie de cette "hétérogénéité non dialectique» de Cornejo Polar.

Victoria Ocampo, traductrice romantique, conçoit la traduction comme un art mimétique. Ocampo, qui se considérait la traductrice «bon marché» de Sur, traduit surtout du théâtre (Graham Greene, Camus, Osborne, Dylan Thomas, entre autres), soit des textes écrits à la première personne. Elle croit que les traductions sont des gants qui s'interposent dans le rapport à l'original et choisit ces textes qui ne s'éloignent pas trop de la façon dont elle-même établit le rapport avec le lecteur (p. 98). À l'inverse de Borges qui, lui, s'attaque à des auteurs (Joyce, Faulkner) qui contredisent sa propre conception de la littérature. Ses stratégies d'adhérence au texte étranger: la non-traduction des noms propres, la conservation des références géographiques, les emprunts et les notes de bas de page. Ces stratégies 
exotisantes, Ocampo les relativise par des expressions courantes qui n'émanent pas d'elle mais «de tous» afin de garantir la lisibilité.

Pour Jorge Luis Borges, la traduction est consubstantielle à la littérature et à son modeste mystère; tout est traduction: lecture, écriture, relecture et réécriture. Une conception vaste et inclusive. Willson commente ainsi nombre d'ouvrages consacrés à Borges avant de se pencher sur quelques textes traduits de Joyce (Ulises), Woolf (Orlando) et Faulkner (Las palmeras salvajes). Elle met au jour les stratégies de décontextualisation, de décotextualisation et de recontextualisation. Elle met l'accent sur les «argentinisations» de Borges et certaines de ses incongruences.

Finalement, José Bianco, le traducteur classique. Pour le définir, Willson cite Borges dans Ficción y reflexión: "Le style de Bianco, comme le cristal ou comme l'air, est invisible. Les mots, pourtant harmonieux, ne s'interposent pas entre l'auteur et les lecteurs» (p. 183, notre traduction). Bianco recherche l'intonation «vraie» mais non « réelle». Il veut sa prose claire, polie, lisse... Il souhaite que le lecteur ne s'aperçoive pas de son effort. C'est en quelque sorte une traduction «fluide», «transparente » et «invisible» qu'il vise. Pour la recevoir, son lecteur devra être conscient de la norme linguistique et, partant, des déviations à cellesci. Même s'il lui arrivait de situer son écriture dans le Río de la Plata, il s'adressait bien davantage à un lectorat hispanophone très large. Patricia Willson examine trois traductions de José Bianco: The Turn of the Screw de Henry James, Les Bonnes de Jean Genet et Malone meurt de Samuel Beckett. Elle y découvre diverses stratégies comme la traduction périphrastique avec ses nombreuses modifications syntaxiques mais compensée par la conservation des références culturelles; la stratégie la plus représentative étant la «sympathie» avec l'auteur, soit l'affinité esthétique, idéologique voire générationnelle, qui engendre une liberté de réécriture "naturelle», ou plutôt naturalisée, du contenu véritable du texte et du style tout aussi véritables de l'auteur (celle des autotraductions notamment). Willson s'arrête assez longuement sur la polémique suscitée par la publication des Bonnes de Genet. Le sujet, la $\mathrm{m}$... de Genet, et le traducteur (anonyme pour la première publication) sont désavoués par Ocampo elle-même. La traduction reparaîtra pourtant onze ans plus tard.

Ces trois traducteurs sont les trois grandes étoiles de la Constellation Sur; ils participent activement à cette entreprise de traduction à la fois démocratique et élitiste qui va marquer d'un renouveau esthétique les littératures argentine, latino-américaine et hispanophone. Ils «traduisent» admirablement cette tension traductionnelle entre l'adéquation au texte source et l'acceptabilité dans la culture réceptrice.

L'aventure céleste se termine sur un chapitre consacré au travail d'importation culturelle des maisons d'édition argentines. Une liste de traductions publiées entre 1933 et 1960 précède les conclusions. Bref, une aventure traductologique pleine de rebondissements, d'éclats et parfois d'échecs, qui n'est autre que l'histoire de la réception des traductions, donc de l'étranger.

On peut regretter la décision de l'auteure de subdiviser sa bibliographie en quatre parties: Bibliographie source, Bibliographie générale, Bibliographie théorique et critique de la traduction et Bibliographie spécifique! Heureusement, les romans d'aventure se passent parfaitement de références bibliographiques. Ce roman de Patricia Willson est à lire d'une traite; il vous transportera au septième ciel...

Pour ceux qui ne voudraient pas en redescendre, je leur proposerais les no 73 et 74 de la revue Ellipse - textes littéraires canadiens en traduction. Le no 73 intitulé «Argentina-Canada» est dirigé par Hugh Hazelton, et le 74, intitulé "Canada-Argentina», par Patrick Imbert (<www.ellipsemag.org $>$ ).

Georges L. BAstin 


\section{RÉFÉRENCES}

Andrade, O. de (1928): «Manifesto Antropófago », Revista de Antropofagia 1-1.

Connejo Polar, A. (1994): Escribir en el aire, Lima, Editorial Horizonte.

García CanClini, N. (1989): Culturas híbridas. Estrategias para entrar y salir de la modernidad, México, Grijalbo.

CÁCEREs Würsig, I. (2004): Historia de la traducción en la Administración y en las relaciones internacionales en España (s. XVI-XIX), [Histoire de la traduction dans l'administration et dans les affaires internationales en Espagne (XVI $\left.{ }^{e}-X I X^{e} s.\right)$ ], Soria, Éditeur Excma, Diputación provincial de Soria, 230 pages.

L'histoire de la traduction reste un mystère pour beaucoup de gens, mais suscite un vif intérêt chez celles et ceux qui veulent élucider, ou simplement souligner, l'apport parfois déterminant des traducteurs à l'évolution d'une société au cours de l'histoire. Le livre Historia de la traducción en la Administración y en las relaciones internacionales en España (s. XVI$X I X)$, d’Ingrid Cáceres Würsig, illustre bien cet intérêt.

L'auteure est professeure d'allemand au Département de traduction et d'interprétation de l'Université européenne de Madrid. Ce livre est l'aboutissement de sa thèse de doctorat soutenue à l'Instituto universitario de Lenguas modernas y Traductores, à l'Universidad Complutens. Il est aussi la chronique du Secrétariat d'interprétation des langues, ou, comme l'indique son titre, l'histoire de la traduction administrative et diplomatique espagnole du $\mathrm{XVI}^{\mathrm{e}}$ au XIX ${ }^{\mathrm{e}}$ siècle.

Cet ouvrage purement référentiel est composé de six chapitres, très clairement subdivisés, à travers lesquels l'auteur nous expose le résultat de ses recherches effectuées dans diverses archives: Archivo Histórico Nacional, Archivo del Ministerio de Asuntos Exteriores et Archivo General de Simanzas, en Espagne.

Le premier chapitre analyse les relations entre la langue et le pouvoir, soit l'administration, l'État et la diplomatie, du $\mathrm{XVI}^{\mathrm{e}}$ au $\mathrm{XIX}^{\mathrm{e}}$ siècle. Les gouvernements, en effet, peuvent, à travers la langue, exercer une forte domination sur un peuple, et même sur d'autres gouvernements, mais ils peuvent aussi s'en servir pour se défendre ou résister à toute tentative de domination. Si la maîtrise de plusieurs langues est indispensable dans les relations diplomatiques, les diplomates ont toujours préféré s'exprimer dans leur langue maternelle, qui incarne leur rang et leur distinction. Cette habitude, illustrée dans l'étude de Cáceres Würsig, s'est maintenue en Espagne jusqu'à nos jours, mais pour d'autres raisons, les hauts fonctionnaires faisant appel à des interprètes et à des traducteurs dans les réunions diplomatiques. Le pouvoir qu'octroie la maîtrise des langues se traduit, pour un diplomate, par une meilleure compréhension non seulement des coutumes et de la culture de ses interlocuteurs, mais aussi de leur façon de penser et d'agir.

Jusqu'au début du $\mathrm{XVI}^{\mathrm{e}}$ siècle, la langue utilisée dans les affaires diplomatiques en Espagne était le latin; c'est ensuite que sont apparus l'italien, puis l'espagnol et le français. Avec l'élargissement des relations diplomatiques entretenues par l'Espagne au xvI ${ }^{\mathrm{e}}$ siècle, les diplomates de métier ont dû maîtriser plusieurs langues et, en l'absence de traducteurs, traduire des documents officiels et diplomatiques. À mesure que la diplomatie espagnole se développait, la présence du traducteur est devenue indispensable et, en 1527, la Secretaría de Interpretación de Lenguas (SIL) a été fondée sous la direction de Diego Gracián de Alderete. Gracián de Alderete, le premier à se voir attribuer le titre de secrétaire en 1536, a exercé ses fonctions de 1527 à 1575. De langue maternelle espagnole, il connaissait le français et l'italien. Il a commencé en tant que secrétaire de l'évêque Francisco de Mendoza, puis, après neuf années au service de l'évêque, est devenu le secrétaire de la SIL. Son travail consistait notamment à traduire et à interpréter des lettres confidentielles et des documents officiels. 